\title{
Modelación del crecimiento individual del callo de hacha Atrina maura (Bivalvia: Pinnidae) a partir de la inferencia multi modelo
}

\author{
Eugenio Alberto Aragón-Noriega \\ Centro de Investigaciones Biológicas del Noroeste, Unidad Sonora. Km 2.35 Camino al Tular, Estero Bacochibampo, \\ Guaymas, Sonora 85454, México; aaragon04@cibnor.mx
}

\author{
Recibido 24-VII-2012. Corregido 20-XI-2012. Aceptado 11-XII-2012.
}

\begin{abstract}
Individual growth modeling of the penshell Atrina maura (Bivalvia: Pinnidae) using a multi model inference approach. Growth models of marine animals, for fisheries and/or aquaculture purposes, are based on the popular von Bertalanffy model. This tool is mostly used because its parameters are used to evaluate other fisheries models, such as yield per recruit; nevertheless, there are other alternatives (such as Gompertz, Logistic, Schnute) not yet used by fishery scientists, that may result useful depending on the studied species. The penshell Atrina maura, has been studied for fisheries or aquaculture supplies, but its individual growth has not yet been studied before. The aim of this study was to model the absolute growth of the penshell $\mathrm{A}$. maura using length-age data. For this, five models were assessed to obtain growth parameters: von Bertalanffy, Gompertz, Logistic, Schnute case 1 and Schnute and Richards. The criterion used to select the best models was the Akaike information criterion, as well as the residual squared sum and $\mathrm{R}^{2}$ adjusted. To get the average asymptotic length, the multi model inference approach was used. According to Akaike information criteria, the Gompertz model better described the absolute growth of $A$. maura. Following the multi model inference approach the average asymptotic shell length was $218.9 \mathrm{~mm}$ (IC 212.3-225.5) of shell length. I concluded that the use of the multi model approach and the Akaike information criteria represented the most robust method for growth parameter estimation of A. maura and the von Bertalanffy growth model should not be selected a priori as the true model to obtain the absolute growth in bivalve mollusks like in the studied species in this paper. Rev. Biol. Trop. 61 (3): 1167-1174. Epub 2013 September 01.
\end{abstract}

Key words: multi model inference, Akaike information criteria, Atrina maura, growth models.

Los biólogos pesqueros enfrentan varios problemas para describir el crecimiento individual de los organismos sujetos a explotación comercial. Primero, deben seleccionar un modelo que mejor describa los datos de longitud a la edad y para esto tienen una gran cantidad de ellos. Los modelos son llamados por los apellidos de quienes los han propuesto, Bertalanffy, Gompertz, Richards entre otros (Schnute 1981). Una vez que se ha decidido por uno, se deben ajustar los parámetros del modelo a utilizar. Los criterios de ajuste más comunes son la minimización de la suma de los residuales al cuadrado y la obtención de la máxima verosimilitud (Haddon 2001). Posteriormente, si se ha elegido más de un modelo, se debe contar con un criterio para usar el más adecuado. Derivado de lo anterior, surgen tres preguntas: ¿Cuál es el modelo que mejor describe el crecimiento individual de la especie bajo estudio? ¿Cómo elijo el modelo? y ¿Qué pretendo resolver con mi estudio de crecimiento individual?

En este caso se decidió modelar el crecimiento individual del callo de hacha Atrina maura (Sowerby 1835). Este molusco bivalvo que habita aguas marinas someras y estuarios en la costa del Pacífico desde Baja California, México hasta Perú (Keen 1971). Es un organismo cuyo crecimiento individual ha sido insuficientemente modelado. Se ha descrito su crecimiento a diferentes temperaturas 
(Leyva-Valencia et al. 2001), su ganancia mensual en talla y peso (Ahumada-Sempoal et al. 2002, Arrieche et al. 2010, Góngora-Gómez et al. 2011), y el único estudio que utilizó un modelo para su crecimiento se limitó al uso del modelo de von Bertalanffy, MCVB (Mendo et al. 2011). La mayoría de los estudios de biología pesquera se concretan en modelar el crecimiento individual con el MCVB porque sus parámetros son la base de otros modelos pesqueros como el de rendimiento por recluta (Zhu et al. 2009).

Para elegir el mejor modelo de crecimiento se utiliza frecuentemente el procedimiento que se denomina inferencia multi-modelo (IMM) y tiene varias ventajas teóricas y prácticas, el cual es un enfoque basado en la teoría de la información. La selección del modelo basado en la teoría de la información es un paradigma relativamente nuevo en ciencias biológicas, y es muy diferente de los métodos tradicionales basados en probar una hipótesis nula (Schnute \& Groot 1992, Burnham \& Anderson 2002, Katsanevakis 2006, 2007, Katsanevakis \& Maravelias 2008). Bajo este enfoque, el análisis de datos es un proceso integrado que involucra:

Seleccionar a priori de un grupo de modelos: Ante la necesidad de ajustar un modelo de forma óptima a un conjunto de datos, es mejor seleccionarlo a partir de un grupo de modelos candidatos (incluidos con base a las características de la especie bajo estudio) en lugar de asumir que existe el mejor modelo y usarlo ajustándolo a los datos.

Seleccionar el mejor modelo: Hay dos categorías de métodos para seleccionar entre modelos candidatos, los empíricos ( $\mathrm{R}^{2}$-ajustada, Bootstrap, validación cruzada, entre otros) y los hipotéticos ( $\mathrm{Cp}$ de Mallows, criterio de información de desviación, predictor final del error, entre otros). Entre estos últimos se encuentra el Criterio de Información de Akaike (AIC, por sus siglas en inglés). El AIC es un estimador simple, asintótico y no desviado de la distancia K-L desarrollado por Akaike
(Burnham \& Anderson 2002) que selecciona el mejor modelo basado en el principio de simplicidad, esto significa que ante dos modelos que se ajustan igual a los datos, se elige el más simple (menor número de parámetros, menor número de supuestos); el modelo con el menor AIC es el que se debe seleccionar. Al calcular el valor de AIC se establece un equilibrio entre la complejidad del modelo y la bondad de ajuste del modelo; es decir, penaliza a un modelo en proporción al número de parámetros. Esto implica que el modelo seleccionado se caracterizará por asegurar que tiene la complejidad y la bondad de ajuste óptimas.

Estimar los parámetros y su precisión: Se realiza a través del proceso llamado IMM que se aplica cuando los datos apoyan a más de un modelo candidato, es decir, cuando la diferencia entre el AIC de un modelo candidato y el AIC menor, es a su vez menor a dos. El proceso implica promediar los parámetros de los modelos apoyados por los datos, en lugar de solo estimar los parámetros del mejor modelo. Esto es una ventaja ya que se llega a inferencias robustas.

El objetivo principal del presente estudio, una vez que se obtuvieron los datos de longitud-edad, fue examinar el crecimiento individual de esta especie por medio de cinco modelos. La decisión del mejor modelo para describir el crecimiento individual de A. maura se determinó siguiendo el criterio AIC y se tomó en cuenta el enfoque IMM para obtener los parámetros de crecimiento individual más adecuados a la especie en estudio.

\section{MATERIALES Y MÉTODOS}

Los modelos candidatos se ajustaron a los datos de longitud-edad del callo de hacha A. maura. Los datos fueron obtenidos de los reportados en la literatura por Góngora-Gómez et al. (2011) derivados del registro de crecimiento en longitud mensual medido durante 16 meses de cultivo en una localidad del Golfo de California $\left(24^{\circ} 30^{\prime} \mathrm{N}-107^{\circ} 48^{\prime} \mathrm{W}\right)$. Los modelos usados fueron: von Bertalanffy (MCVB), 
Gompertz, logístico (Ricker 1975) el caso uno del modelo desarrollado por Schnute (1981) y Schnute \& Richards (1990), los cuales se describen a continuación:

El MCVB que se representa por la ecuación:

$$
\mathrm{L}_{(\mathrm{t})}=\mathrm{L}_{\infty}\left(1-\mathrm{e}-\mathrm{k}\left(\mathrm{t}-\mathrm{t}_{0}\right)\right)
$$

El modelo logístico:

$$
\mathrm{L}_{(\mathrm{t})}=\mathrm{L}_{\infty}\left(1-\mathrm{e}-\mathrm{k}_{2}\left(\mathrm{t}-\mathrm{t}_{1}\right)\right)^{-1}
$$

El modelo de Gompertz descrito por la ecuación:

$$
\left.\mathrm{L}_{(\mathrm{t})}=\mathrm{L}_{\infty} \mathrm{e}^{\left(-1 / \mathrm{k}_{3}\right) \mathrm{e}-\mathrm{k}_{3}\left(\mathrm{t}-\mathrm{t}_{2}\right)}\right)
$$

El modelo de Schnute \& Richards (1990) expuesto por la siguiente ecuación:

$$
\mathrm{L}_{(\mathrm{t})}=\mathrm{L}_{\infty}\left(1+\delta \mathrm{e}^{\mathrm{k}_{4} \mathrm{tv}}\right)^{1 / \gamma}
$$

Para todos estos modelos los parámetros evaluados se describen como:

$L_{(t)} \quad$ longitud a la edad $t$

$t \quad$ edad a la longitud $L_{(t)}$

$L_{\infty} \quad$ longitud promedio de un organismo muy viejo (Longitud asintótica)

$k \quad$ determina la rapidez para alcanzar $L_{\infty}$ (Parámetro de curvatura)

$t_{0} \quad$ edad hipotética a la cual la longitud del organismo es cero

$k_{2} \quad$ tasa relativa de crecimiento

$t_{1}$ punto de inflexión de la curva sigmoidea

$t_{2} \quad$ es $\frac{\operatorname{Ln} \lambda}{\mathrm{k} 3}$

$\lambda$ es la tasa de crecimiento relativa inicial teórica a la edad cero (unidades de año ${ }^{-1}$ )

$k_{3} \quad$ es la tasa de decaimiento exponencial de la tasa de crecimiento relativa (unidades de año ${ }^{-1}$ )

$\delta, v, \gamma$ son parámetros adimensionales

$k_{4} \quad$ tiene unidades de año ${ }^{-1}$

El modelo de Schnute adquiere cuatro formas matemáticas. En este estudio se usó el caso uno cuando $\lambda \neq 0, \rho \neq 0$,

$$
L(t)=\left[1^{\rho}+\left(\delta^{\rho} \mid-1^{\rho}\right) 1-e-\lambda\left(t-\tau_{1) /} 1-e-\lambda\left(\tau_{2}-\tau_{1}\right)\right]^{1 / \rho}\right.
$$

Donde:

$\tau_{1} \quad$ es la menor edad en el set de datos

$\tau_{2}$ es la mayor edad en el set de datos

$\lambda$ es una tasa constante relativa de la tasa relativa de crecimiento. Es un parámetro a estimar

$\rho$ es una tasa de incremento relativa de la tasa relativa de crecimiento. Es un parámetro a estimar

1 tamaño a la edad $\tau_{l}$. Es un parámetro a estimar

$\delta$ tamaño a la edad $\tau_{2}$. Es un parámetro a estimar

Para el modelo de Schnute que no calcula el parámetro $L_{\infty}$ en su estimación directa se debe aplicar la siguiente ecuación:

$$
\mathrm{L} \infty=\left[\mathrm{e}^{\lambda \tau 2 \delta \rho}-\mathrm{e} \lambda \tau 1 \rho / \mathrm{e}^{\lambda \tau 2}-\mathrm{e}^{\lambda \tau 1}\right]^{1 / \rho}
$$

Según lo propuesto por Schnute (1981) y cuyos parámetros ya fueron definidos anteriormente.

Los datos se ajustaron a cada uno de los diferentes modelos de crecimiento antes mencionados con la función de máxima verosimilitud con el algoritmo de Newton (Haddon 2001).

$$
\mathrm{LL}=\mathrm{n} / 2\left(\mathrm{~L}_{\mathrm{n}} 2 \pi+2 \mathrm{~L}_{\mathrm{n}} \sigma+1\right)
$$

considerando

$$
\sigma=\text { raíz }\left(\mathrm{Lt}_{\text {observado }}-\mathrm{Lt}_{\text {calculado }}\right)^{2 / n}
$$

Decisión del mejor modelo: La selección del modelo se llevó a cabo a través de una forma corregida del AIC (Burnham \& Anderson 2002). Esta corrección se realiza si la razón $\mathrm{n} / \mathrm{k}$ es menor a 40 . Para eso, se toma en cuenta el modelo con la mayor cantidad de parámetros y se asume que las desviaciones están normalmente distribuidas con varianza constante. El modelo con el menor valor de $\mathrm{AIC}_{\mathrm{c}}\left(\mathrm{AIC}_{\mathrm{c}, \min }\right)$ fue el seleccionado como el mejor.

$$
\begin{gathered}
\mathrm{AIC}_{\mathrm{c}}=\mathrm{AIC}+(2 \mathrm{k}(\mathrm{k}+1) / \mathrm{n}-\mathrm{k}-1) \\
\mathrm{AIC}=2 \mathrm{LL}+(2 \mathrm{k})
\end{gathered}
$$


Donde:

$L L$ es la verosimilitud logarítmica negativa

$n \quad$ es el número de observaciones

$k \quad$ es el número de parámetros en el modelo

Se calcularon las diferencias entre los $\mathrm{AIC}_{\mathrm{c}}$ de cada modelo y el $\mathrm{AIC}_{\mathrm{c}}$ menor o mínimo:

$$
\Delta_{\mathrm{i}}=\mathrm{AIC}_{\mathrm{c}, \mathrm{i}}-\mathrm{AIC}_{\mathrm{c}, \text { mínimo }}
$$

Además de la plausibilidad (el peso de la evidencia a favor del modelo $i$ ) a través de la "ponderación del criterio de información Akaike" como se describe a continuación:

$$
\mathrm{W}_{\mathrm{i}}=\mathrm{e}\left(-0.5 \Delta_{\mathrm{i}}\right) / \Sigma\left(-0.5 \Delta_{\mathrm{i}}\right)
$$

Siguiendo un enfoque de inferencia múltimodelo, se determinó $L_{\infty}$ "promedio" de todos modelos candidatos y usando los correspondientes $W_{i}$ para ponderar.

$$
\overline{\mathrm{L}}_{\infty}=\Sigma \mathrm{W}_{\mathrm{i}} \overline{\mathrm{L}}_{\infty, \mathrm{i}}
$$

De igual manera, se calculó el error estándar y el intervalo de confianza al $95 \%$ de la longitud asintótica promedio:

$$
\overline{\mathrm{L}}_{\infty}= \pm \mathrm{t}_{\mathrm{g} 1,0.975} \text { Error Estándar }\left(\overline{\mathrm{L}}_{\infty}\right)
$$

Donde:

$$
\text { Error Estándar }\left(\overline{\mathrm{L}}_{\infty}\right)=\Sigma \mathrm{W}_{\mathrm{i}}\left(\operatorname{var}\left(\overline{\mathrm{L}}_{\infty, \mathrm{i}} \mid \mathrm{g}_{1}\right)+\left(\overline{\mathrm{L}}_{\infty, \mathrm{i}}-\overline{\mathrm{L}}_{\infty}\right)^{2}\right)^{1 / 2}
$$

\section{RESULTADOS}

La figura 1 presenta los datos observados y las curvas resultantes para cada uno de los cinco modelos utilizados, los cuales describen trayectorias similares por lo que a simple vista no se observa cuál describe mejor los datos y aparentemente todos pueden seleccionarse como buenos. El cuadro 1 muestra jerárquicamente el orden de los modelos. El Gompertz obtuvo el menor valor de AIC (111.8) mientras que el MCVB y el Schnute \& Richards mostraron los mayores índices (119.4 y 119.8) El valor de ponderación $\left(W_{i}\right)$. para el Gompertz fue de $79.75 \%$, y menos de $2 \%$ tanto para el MCVB como al de Schnute \& Richards.

La longitud asintótica $L_{\infty}$ promedio encontrada, siguiendo el enfoque de IMM, fue de 218.9mm (IC 212.3-225.5) de longitud de concha (Cuadro 1). Cabe mencionar que la IMM lo que propone para obtener el valor promedio no es el promedio aritmético de todas $L_{\infty}$ estimadas en cada modelo. En su lugar, es la sumatoria de cada $L_{\infty}$ multiplicada por su valor de ponderación $\left(W_{i} \%\right)$, y con esto se otorga el peso de cada modelo a la estimación de la $L_{\infty}$ promedio. La mayor $L_{\infty}$ estimada fue con el MCVB con un valor de $243.9 \mathrm{~mm}$ (IC 239.6248.1) de longitud de concha (Cuadro 1).

Los valores para los criterios de selección de modelos se presentan en el cuadro 2 . Se observa que tanto la SRC, $\mathrm{R}^{2}$ ajustada y AIC indican al Gompertz (617, 0.9986 y 111.18,

CUADRO 1

Valores de AIC, $\Delta_{\mathrm{i}}, W_{i}$ y $L_{\infty}$ obtenidos de los modelos ajustados con los datos promedios de longitud a la edad, para Atrina maura del Golfo de California

TABLE 1

Values of AIC, $\Delta_{\mathrm{i}}, W_{i}$ and $L_{\infty}$ calculated for the models fitted to the averaged length at age data of Atrina maura from the Gulf of California

\begin{tabular}{lcccccccc} 
& $\mathrm{k}$ & $\mathrm{AIC}$ & $\boldsymbol{\Delta}_{\mathrm{i}}$ & $\mathrm{W}_{\mathrm{i}}(\%)$ & $L_{\infty}$ & $\begin{array}{c}\text { Error } \\
\text { estándar }\end{array}$ & \multicolumn{2}{c}{ Intervalo de confianza 95\% } \\
Gompertz & 3 & 111.8 & 0.00 & 79.75 & 219.12 & 1.55 & 215.8 & 222.5 \\
Logístico & 3 & 115.3 & 3.41 & 14.47 & 212.02 & 1.73 & 208.3 & 215.8 \\
Schnute & 4 & 117.8 & 6.00 & 3.97 & 228.23 & 1.67 & 224.6 & 231.9 \\
MCVB & 3 & 119.4 & 7.57 & 1.81 & 243.88 & 1.97 & 239.6 & 248.1 \\
Schnute \& Richards & 5 & 119.8 & 8.00 & 1.46 & 219.02 & 1.55 & 215.6 & 222.4 \\
Promedio & & & & & 218.90 & 3.13 & 212.3 & 225.5 \\
\hline
\end{tabular}




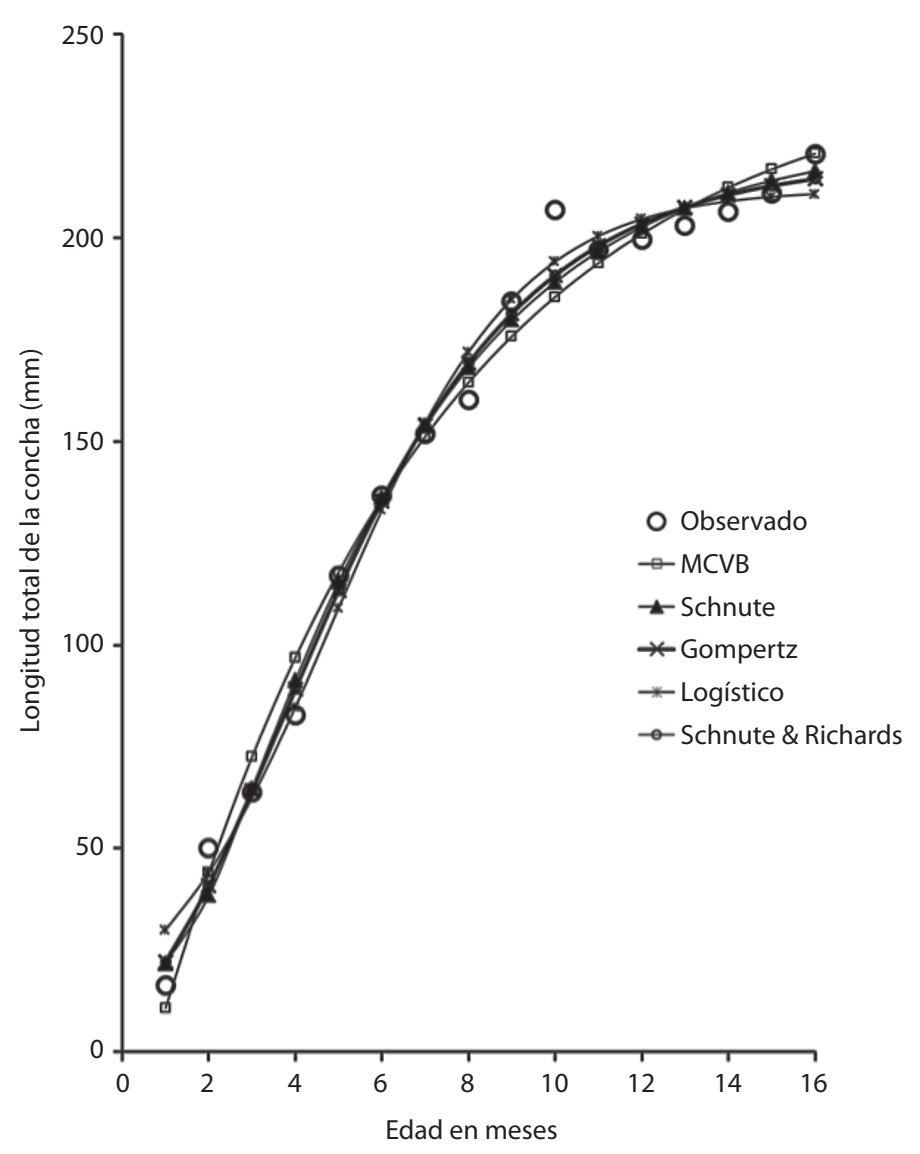

Fig. 1. Curvas de crecimiento generadas para los cinco modelos aplicados a datos de longitud a la edad promedio de Atrina maura del Golfo de California.

Fig. 1. Growth curves fitted for five models to the averaged length at age data of Atrina maura from the Gulf of California.

respectivamente) como el mejor. Es importante también señalar que los criterios SRC y $\mathrm{R}^{2}$ ajustada seleccionan al modelo de Schnute \& Richards como el mejor modelo y con los mismos valores que al modelo de Gompertz, mientras que AIC coloca al modelo de Schnute $\&$ Richards en el último lugar ya que le otorga el valor más alto.

\section{DISCUSIÓN}

En estudios anteriores, los parámetros de crecimiento de otras especies de almejas se calcularon utilizando un algoritmo basado en el método de mínimos cuadrados (Hoffmann et al. 2000, Campbell \& Ming 2003, CalderónAguilera et al. 2010). En este estudio, en su lugar se utilizó como criterio la máxima verosimilitud, encontrando que el uso de ésta representa una mejor solución para estimar adecuadamente los parámetros de los modelos de crecimiento individual tal como lo propuso Katsanevakis (2006) para otros modelos, ya en general la máxima verosimilitud es una prueba más robusta.

Al comparar entre varios modelos de crecimiento individual, se utilizaron como criterios de selección el valor de $\mathrm{R}^{2}$ ajustada, la suma de residuos al cuadrado SRC y el AIC. La selección de modelos basado en la teoría 
CUADRO 2

Valores de los tres diferentes criterios de selección de modelos

TABLE 2

Values for three different model selection criteria

\begin{tabular}{lccc}
\multicolumn{1}{c}{ Modelo } & SRC & $\mathrm{R}^{2}$ ajustada & AIC \\
Gompertz & 617 & 0.9986 & 111.8 \\
Schnute \& Richards & 617 & 0.9986 & 119.8 \\
Schnute & 715 & 0.9983 & 117.8 \\
Logístico & 764 & 0.9982 & 115.3 \\
MCVB & 990 & 0.9977 & 119.4 \\
\hline
\end{tabular}

$\mathrm{SRC}=$ Suma de residuales al cuadrado.

$\mathrm{AIC}=$ Criterio de Información de Akaike.

de la información ha sido recomendado como una alternativa mejor y más robusta que los enfoques tradicionales (Katsanevakis 2006, Cerdenares-Ladrón de Guevara et al. 2011). Las ventajas de utilizar el AIC son que los modelos pueden ordenarse jerárquicamente según su ajuste a los datos, y la obtención de su parámetro promedio. Sin embargo, el parámetro promedio deseado (por ejemplo, longitud asintótica), se estima obteniendo la ponderación de Akaike (Burnham \& Anderson 2002); es decir, la contribución de cada parámetro es ponderada antes de obtener el promedio del parámetro deseado.

El uso de $\mathrm{R}^{2}$ ajustada y la suma de cuadrados en la selección de modelos no toman en cuenta la estructura que mejor se ajusta, sino que la decisión de seleccionarlo se basa en la complejidad del mismo modelo. De hecho, $\mathrm{R}^{2}$ ajustada y la suma de cuadrados tienden a seleccionar el modelo más complejo (Zhu et al. 2009). En contraste, el AIC selecciona el más simple, porque penaliza la adición de más parámetros al modelo. En el presente estudio, los criterios para la selección del modelo, es decir, $\mathrm{R}^{2}$ ajustada, la SRC y el AIC, todos indicaron el mismo modelo (Gompertz) como el mejor. Cabe señalar que sólo Schnute \& Richards incluye cinco parámetros, mientras que el de Gompertz utiliza tres parámetros. Probablemente por ello, tanto la $\mathrm{R}^{2}$ ajustada como la suma cuadrada de residuos seleccionó el modelo de Schnute \& Richards, como se esperaba, basado en que estos dos criterios estadísticos seleccionarían el modelo más complejo debido a que la complejidad aumenta el valor de $\mathrm{R}^{2}$ ajustada y disminuye la SRC (Zhu et al. 2009). Con el modelo de Schnute \& Richards se cumplió el principio de que a mayor complejidad tiende a aumentar el valor de $\mathrm{R}^{2}$ ajustada y a disminuir la SRC. De igual forma estos dos enfoques seleccionaron al Gompertz como el mejor modelo para representar el crecimiento de A. maura cumpliendo también el principio de que seleccionarían al modelo con mayor ajuste.

Según el AIC, se encontró el modelo de Gompertz como el mejor, mientras que el menos compatible fue el de Schnute \& Richards. Aunque el MCVB es el más estudiado y comúnmente aplicado entre todos modelos de longitud a la edad, su uso como el único modelador de crecimiento no está bien soportado. Con respecto a otros estudios usando los $\mathrm{AIC}$, Baer et al. (2011) concluyó que el MCVB no es el modelo óptimo para calcular el crecimiento del pez plano (Psetta maxima). Resultados similares fueron encontrados por Flores et al. (2010) en el erizo de mar (Loxechinus albus). Una crítica completa de la aplicación $a$ priori del MCVB en otras especies de callo de hacha como Pinna nobilis se puede consultar en Katsanevakis (2007) en donde se mencionan Gompertz y Logístico como los mejores modelos para describir el crecimiento absoluto especialmente en moluscos bivalvos. En el presente estudio se encontró que tanto Gompertz como Logístico fueron seleccionados como los mejores modelos (en ese orden jerárquico) ya que juntos completan una ponderación del $94.22 \%$ dejando con el restante porcentaje a los otros tres modelos.

Está claro que este nuevo enfoque estadístico basado en la teoría de la información se ha vuelto cada vez más popular en la inferencia matemática, pero es muy reciente en estudios biológico-pesqueros, donde ha sido usado al menos durante una década. A pesar de ello, Mundry (2011) sugiere usarlo con precaución en estudios de Ecología y propone una mezcla 
de la utilización de pruebas de hipótesis nula y criterios de teoría de información en circunstancias específicas. Por lo tanto, se espera que en los estudios pesqueros (especialmente en especies acuáticas tropicales) el uso de AIC se convierta en una herramienta común en la selección de modelos, pero todavía se pueden utilizar pruebas de hipótesis nula con suficiente justificación.

En el presente trabajo, se eligió utilizar la IMM para seleccionar el mejor ajuste del parámetro $L_{\infty}$. Se encontró que la longitud asintótica de la concha de $A$. maura, fue de $218.9 \mathrm{~mm}$, con un intervalo al $95 \%$ de confianza, de 212.3-225.5mm. Esto mediante el promedio de la longitud asintótica que se estimó en los cinco modelos utilizados. Hasta ahora no se puede comparar este resultado porque ningún estudio previo de crecimiento de A. maura ha reportado el valor de longitud asintótica. Es importante señalar que Mendo et al. (2011) no calcularon la longitud asintótica de la concha de $A$. maura, utilizando el MCVB, en la región del Pacífico mexicano y optaron por fijarla en $335 \mathrm{~mm}$ como representante del organismo más longevo y sin aclarar si fue en su zona de estudio ya que según sus propios reportes el organismo más grande fue de $218 \mathrm{~mm}$ de longitud de concha. Góngora-Gómez et al. (2011) registraron el organismo más grande con $220.5 \mathrm{~mm}$. Ahumada-Sempoal et al. (2002) reportaron la estructura de tallas de $A$. maura, en una región más tropical del Pacífico mexicano $\left(16^{\circ} 11^{\prime} \mathrm{N}\right.$ $98^{\circ} 05^{\prime} \mathrm{W}$ ) y mencionan que el promedio fue de $181 \mathrm{~mm}$ y el máximo $285 \mathrm{~mm}$. La conclusión de este estudio es que tanto la IMM como el AIC representan las herramientas más sólidas para evaluar parámetros de crecimiento individual en la especie estudiada.

\section{AGRADECIMIENTOS}

Este estudio forma parte del programa de ordenamiento pesquero del noroeste de México. El autor recibió financiamiento del proyecto CIBNOR EP0.01. Se agradece a los revisores anónimos que hicieron importantes mejoras al manuscrito.

\section{RESUMEN}

El molusco bivalvo Atrina maura (Sowervy 1835) habita aguas marinas someras y estuarios en la costa del Pacífico desde Baja California, México hasta Perú. Es un organismo cuyo crecimiento individual ha sido insuficientemente modelado. El objetivo del estudio fue modelar su crecimiento individual absoluto mediante el uso de datos de longitud a la edad obtenidos de una región del Golfo de California. Los parámetros fueron obtenidos con el uso de cinco modelos de crecimiento; von Bertalanffy, Gompertz, Logístico, Caso 1 de Schnute y Schnute \& Richards. Se seleccionó el mejor modelo usando el criterio de información de Akaike, AIC, pero se calcularon también la suma de residuos al cuadrado y la $\mathrm{R}^{2}$ ajustada. Para obtener la longitud asintótica se siguió el enfoque de inferencia multi modelo IMM. Según el AIC el modelo que mejor describe el crecimiento absoluto de A. maura es el de Gompertz y colocó en cuarto lugar jerárquico el de von Bertalanffy. La longitud asintótica promedio encontrada, siguiendo la IMM, fue de 218.9mm (IC 212.3-225.5) de longitud de concha. Se concluye que tanto la IMM como el AIC representan las herramientas más sólidas para evaluar parámetros de crecimiento individual de $A$. maura y que el modelo de crecimiento de von Bertalanffy no debe ser seleccionado a priori como la opción para describir el crecimiento individual en moluscos bivalvos como la especie estudiada.

Palabras clave: inferencia multi modelo, criterio de información de Akaike, Atrina maura, modelos de crecimiento.

\section{REFERENCIAS}

Ahumada-Sempoal, M., S. Serrano-Guzmán \& N. RuizGarcía. 2002. Abundancia, estructura poblacional y crecimiento de Atrina maura (Bivalvia: Pinnidae) en una laguna costera tropical del Pacífico mexicano. Rev. Biol. Trop. 50: 1091-1100.

Arrieche, D., A.N. Maeda-Martínez, J.A. Farías-Sánchez \& P.E. Saucedo. 2010. Desempeño biológico del callo de hacha Atrina maura y el mejillón Mytella strigata en diferentes regímenes de flujo de agua. Cienc. Mar. 36: 237-248.

Baer, A., C. Schulz, I. Traulsen \& J. Krieter. 2011. Analysing the growth of turbot (Psetta maxima) in a commercial recirculation system with the use of three different growth models. Aquacult. Int. 19: 497-511.

Burnham, K.P. \& D.R. Anderson. 2002. Model selection and multi-model inference: a practical informationtheoretic approach. Springer, Nueva York, EE.UU.

Calderón-Aguilera, L.E., E.A. Aragón-Noriega, C.M. Hand \& V.M. Moreno-Rivera. 2010. Morphometric relationships, age, growth and mortality of the geoduck clam Panopea generosa, along the Pacific coast 
of Baja California, Mexico. J. Shellfish Res. 29: 319-326.

Campbell, A. \& M.D. Ming. 2003. Maturity and growth of the Pacific geoduck clam, Panopea abrupta, in Southern British Columbia, Canada. J. Shellfish Res. 22: 85-90.

Cerdenares-Ladrón de Guevara, G., E. Morales-Bojórquez \& R. Rodríguez-Sánchez. 2011. Age and growth of the sailfish Istiophorus platyoterus (Istiophoridae) in the Gulf of Tehuantepec, Mexico. Mar. Biol. Res. 7: 488-499.

Flores, L., B. Ernst \& A.M. Parma. 2010. Growth pattern of the sea urchin, Loxechinus albus (Molina, 1782) in southern Chile: evaluation of growth models. Mar. Biol. 157: 967-977.

Góngora-Gómez, A.M., M. García-Ulloa, A.L. Domínguez-Orozco \& J.A. Hernández-Sepúlveda. 2011. Crecimiento del callo de hacha Atrina maura (Sowerby, 1835) (Bivalvia: Pinnidae) cultivado a diferentes densidades. Avanc. Invest. Agrop. 15: 79-94.

Haddon, M. 2001. Modelling and quantitative methods in fisheries. Chapman and Hall/CRC, Florida, EE.UU.

Hoffmann, A., A. Bradbury \& C.L. Goodwin. 2000. Modelling geoduck, Panopea abrupta (Conrad 1849) population dynamics I. Growth. J. Shellfish Res. 19: 57-62.

Katsanevakis, S. 2006. Modelling fish growth: Model selection, multi-model inference and model selection uncertainty. Fish. Res. 81: 229-235.

Katsanevakis, S. 2007. Growth and mortality rates of the fan mussel Pinna nobilis in Lake Vouliagmeni (Korinthiakos Gulf, Greece): a generalized additive modeling approach. Mar. Biol. 152: 1319-1331.
Katsanevakis, S. \& D. Maravelias. 2008. Modelling fish growth: multi-model inference as a better alternative to a priori using von Bertalanffy equation. Fish and Fish. 9: 178-187.

Keen, A.M. 1971. Sea shells of Tropical West America. Marine mollusks from Baja California to Peru. Stanford University, California, EE.UU.

Leyva-Valencia, L., A.N. Maeda-Martinez, M.T. Sicard, I. Roldan \& M. Robles-Mungaray. 2001. Halotolerance, upper thermotolerance, and optimum temperature for growth of the penshell Atrina maura (Sowerby, 1835) (Bivalvia: Pinnidae). J. Shellfish Res. 20: 49-54.

Mendo, T., V. Koch, M. Wolff, F. Sínsel \& C. Ruiz-Verdugo. 2011. Feasibility of intertidial bottom culture of the penshell Atrina maura in Bahía Magdalena, Baja California Sur, México. Aquaculture 314: 252-260.

Mundry, R. 2011. Issues in information theory-based statistical inference a commentary from a frequentist's perpective. Behav. Ecol. Sociobiol. 62: 57-68.

Ricker, W.E. 1975. Computation and interpretation of biological statistics of fish populations. Fish. Res. Board Can Bull. 191: 1-382.

Schnute, J. 1981. A versatile growth model with statistically stable parameters. Can. J. Fish. Aquat. Sci. 38: 1128-1140.

Schnute, J. \& K. Groot. 1992. Statistical analysis of animal orientation data. Anim. Behav. 43: 15-33.

Schnute, J. \& L.J. Richards. 1990. A unified approach to the analysis of fish growth, maturity, and survivorship data. Can. J. Fish. Aquat. Sci. 47: 24-40.

Zhu, L., L. Li \& Z. Liang. 2009. Comparison of six statistical approaches in the selection of appropiate fish growth models. Chinese J. Oceanol. Limnol. 27: 457-467. 